\title{
Development and Performance Evaluation of Dilution System for Diagnosis Systems with Saliva
}

\author{
Yuji Jou $^{1}$, Keisuke MORISHIMA ${ }^{2}$, Yuji FURUKAWA ${ }^{1}$ \\ ${ }^{1}$ Department of Management of Technology, Tokyo University of Agriculture and Technology, Japan, \\ 50006311018@st.tuat.ac.jp \\ ${ }^{2}$ Department of Mechanical systems engineering, Tokyo University of Agriculture and Technology, Japan, \\ morisima@cc.tuat.ac.jp
}

\begin{abstract}
:
In order to use saliva for human stress diagnosis, ELISA (Enzyme-Linked ImmunoSorbent Assay) is most promising, when saliva must be diluted in purified water from about $10^{1}$ to $10^{4}$ times corresponding to the applied stress markers. A new dilution system with 4 diluting ladders in which each step has 10 times dilutive capability is developed, hence, can realize $10^{1}$ to $10^{4}$ step-likely. Its performance was tested by having mixed a fluorescent material in pure water and checked its change of intensity, and it is verified that $+-20 \%$ error for the $10^{4}$ time dilution target is kept.
\end{abstract}

Keywords: human stress, saliva, micro channel, dilution system

\section{Introduction}

In modern society, human's mental and physical stresses became something of a topic of conversation and several methods to diagnose human's stress such as an interview or physical diagnoses using blood, urine and saliva have been developed. The interview method is commercially applied today, however, medical doctors claim that it depends much on a decision of each doctor and sometimes difficult to answer objectively, so wish strongly to develop any physical inspective method [1]. Unfortunately, the physical diagnoses are now on the test and not practically used due to its complexity of procedures and expensive unit cost. Among them, the method to use saliva for stress checker may be preferred for its easiness of sampling with lower burden on patients [2].

The principle to diagnose human's stress by checking some markers involved in saliva is known medically, that is, ELISA (Enzyme-Linked Immunosorbent Assay) based diagnosis is applied, in which, how much protein is bound by an antibody can be determined. In these examples, how much of a particular antibody is present in an individual's saliva is checked. As the antibodies, three markers shown in Table 1 are known suitable for the human's stress diagnosis. In practice, IgA (Immunoglobulin A ) and cortisol were chosen as the stress markers and EGF (Epithelial Growth Factor ) was used for a cell aging marker in our previous work [3].

However, in the normal ELISA systems using 96 wells, much amount of reagent as well as much sample is generally required, hence, increase its test cost inevitably and several hours are usually needed before the reagent reacts with the sample. In order to overcome these defects, application of $\mu$-TAS is promising and a prototype has been developed by our TAMA consortium project [3]. The similar method is reported by another Japanese $R \& D$ group [4].

However, there remains a problem to have to dilute three markers with so much different dilution rates, that is, cortisol must be 5 times, EGF 100 times, and IgA 5000 times in pure water respectively. To overcome this, application of $\mu$-TAS is promising and a novel dilution system [3] consisting of a Y shaped micro channel was proposed, from each two inlets of which minimum required amount of saliva and solution liquid are pushed out by a Braille-device and mixed in a jointed flow, however, it could only verify the principle of dilution [5-6].

In the present paper, a 4 ladder dilution system was designed and examined, in which each ladder can dilute saliva up to 10 times, hence saliva is diluted $10^{2}, 10^{3}$, and $10^{4}$ times in progress with laddering. To visualize a flow, a fluorescent solution was sent into the micro-channel instead of saliva, with which the system's performance was evaluated.

\begin{tabular}{|c|c|c|}
\hline $\begin{array}{c}\text { Biological } \\
\text { marker }\end{array}$ & Target & Required dilution rate \\
\hline IgA & Stress & $5000 \sim 10000$ \\
\cline { 1 - 1 } \cline { 3 - 3 } Cortisol & Cell aging & $5 \sim 10$ \\
\hline EGF & $100 \sim 200$ \\
\hline
\end{tabular}

Table 1: Biological makers and their dilution rates This table represents three makers desirable for the diagnosis system using saliva. The makers must be diluted appropriately.

\section{Principle and Design}

The dilution system was co-developed with Prof. Takayama at University of Michigan [7-8] originally. The dilution system consists of a Braille device and a PDMS-made chip. The Braille display provides a grid of micro actuator pins. These pins are actuated vertically by 
piezoelectric bimorphs that can add to $280 \mathrm{kPa}$ of localized pressure. The Braille pins were controlled using Visual Basic. The chip is fabricated by lithography of PDMS. The mold of micro-channel was also fabricated by photolithography, to which PDMS was poured. Finally, PDMS membrane is adhered to the backside of micro-channel of chip. In the first trial, the cross-section of micro-channel was square and that resulted in incomplete shut of channel when the top membrane was pushed by Braille device and closed the channel by elastic deformation. In the current research, the defect was improved that the bell shaped channel (Fig.2) was fabricated by a special photolithography with scattered light and could shut the channel completely. In details, the micro-channel of PDMS chip placed on those Braille pins,

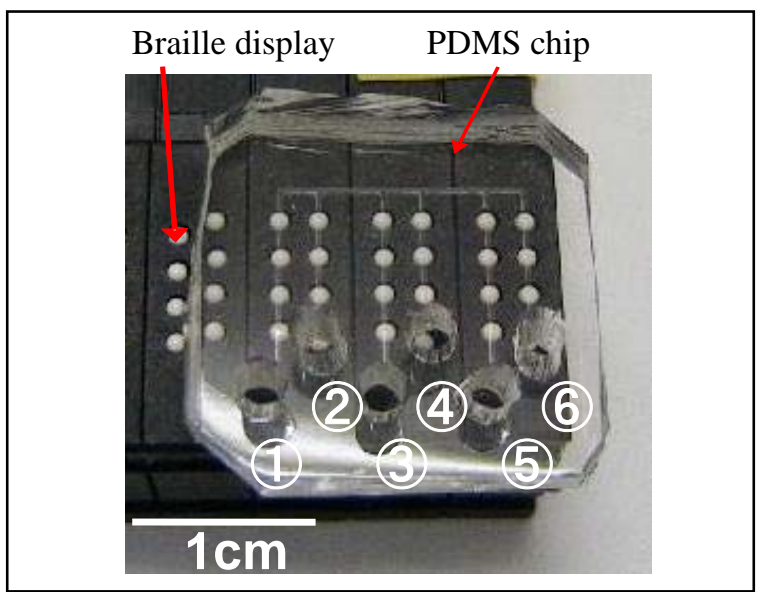

Figure 1: The Schematic of dilution system. It consists of the Braille device and the PDMS chip with six inlets.

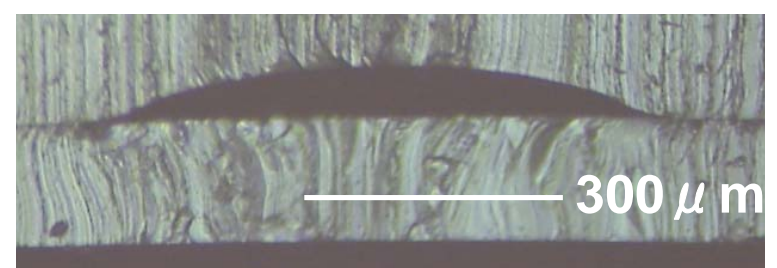

Figure 2: The cross-section of microchannel made by photolithography.

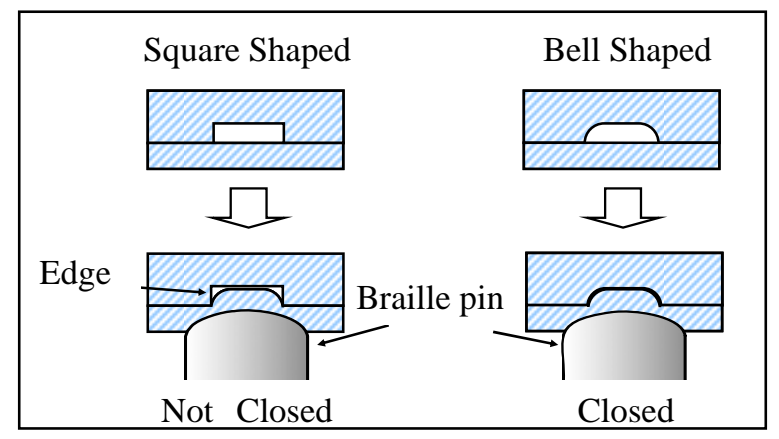

Figure 3: The comparison of opening and shutting of microchannel section shapes. The rectangular cross-section is difficult to shut by extrusion. However, the bell shaped one is easy for shut. with the surface of membrane below as shown in Fig.3. The membrane deforms elastically by the Braille pin extrusion, and the channel is opened and closed.

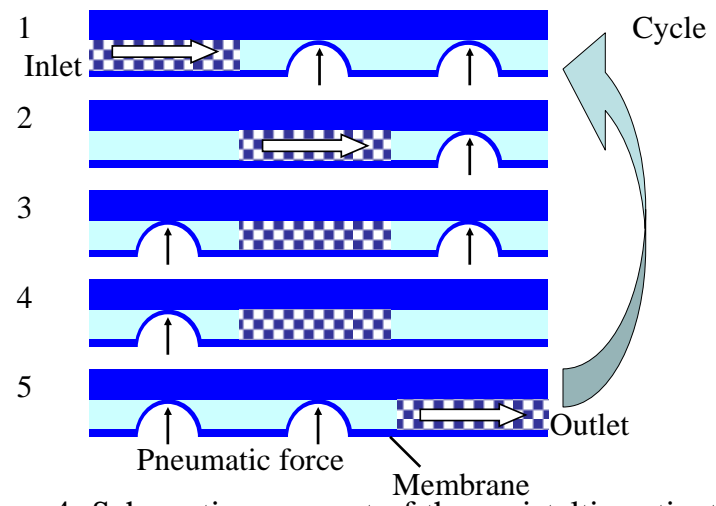

Figure 4: Schematic represent of the peristaltic activation of three sets of brille pins to pump the fluids to flow in specific direction and to regulate the fluid flow rate.

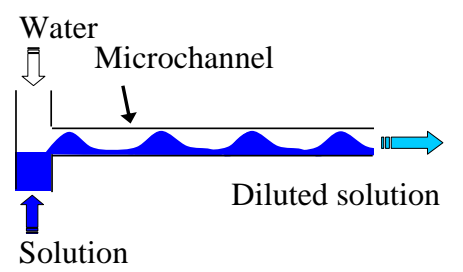

Figure 5: Principle of dilution with T shaped channel. The dilution rate is controlled by frequency ratio of the solution sending from two inlets.
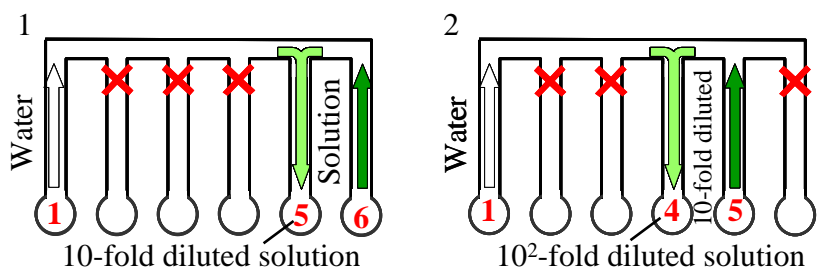

3

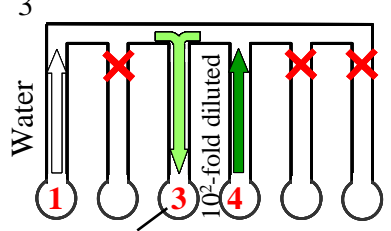

4

$10^{3}$-fold diluted solution

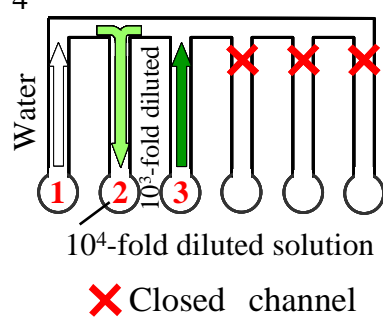

Figure 6: The principle of 4ladder dilution with six $\mathrm{T}$ shaped microchannels. Water is put in inlet 1 , the solution is put in inlet 6 , and water: solution is 9:1, and these liquid is send. And the 10 -fold diluted solution is produced in inlet 5 . Next, dilution similarly is repeated at 4times in total, and dilute at $10^{2}, 10^{3}, 10^{4}$ fold.

Fig.4 shows a schematic representation of peristaltic motion of PDMS membranes which is driven by an external Braille pins, consequently, the flow rate is proportional to the peristaltic cycle.

The principle of dilution is shown in Fig.5, where a certain solution and water are pushed out alternately from the two inlets and mixed in the joined channel, hence, the dilution rate can be controlled by the ratio of sending their 
amounts from two inlets. In practice, it can dilute up to 10 times, that means to push out the solution once while the water 10 times taking optimum reaction time into account. Fig.6 shows the ladder dilution systems where the basic dilution unit is added 4 ladders. In this way, it becomes possible to dilute $10^{\mathrm{N}}$ times if the ladder consists of $\mathrm{N}$ layers.

\section{Experimental Method}

\subsection{Measurement of flow rate}

The flow rate of micro-channel when pushed by Braille pins is measured by two methods. As an average flow rate, the volume change of dissolved liquid which was led to the attached silicon tube with $1 \mathrm{~mm}$ diameter was measured with a specified time as shown in Fig. 7. On the other hand, a precise flow rate inside of micro-channel is investigated from an image of fluorescent solution by microscope as also shown in Fig.7. In both tests, the effect of viscosity on a flow rate is checked by changing the percentage of glycerin for water.

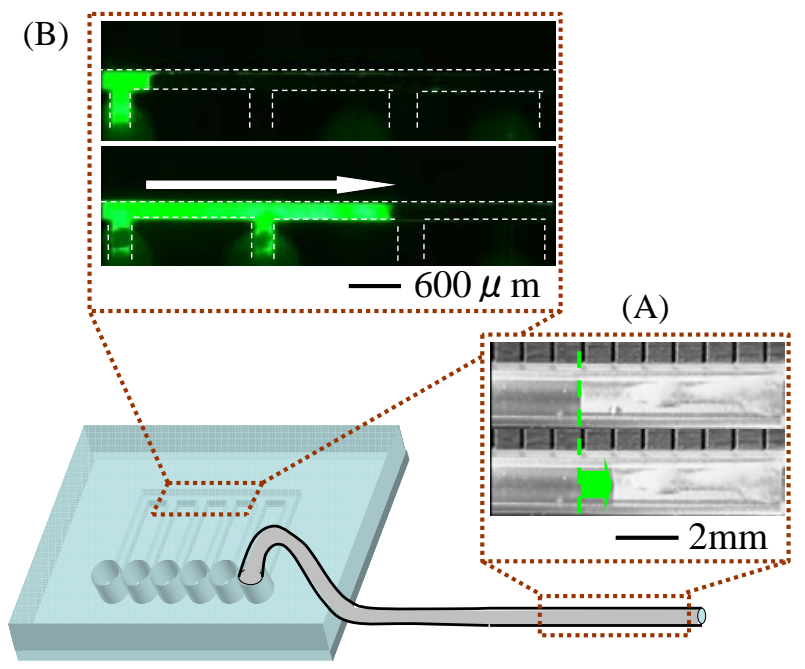

Figure 7: The flow rate measured from displacement of the gas liquid surface. (A)Measurement in silicon tube (inside diameter is $1 \mathrm{~mm}$ ) connected with microchannel inlet. (B) Measurement in microchannel. This image was observed with a fluorescence microscope and fluorescence solution. The cross-section area of the channel used in measurement is about $84000 \mu^{2}$.

\subsection{Measurement of dilution rate}

A reference liquid solved a known amount of Fluorescent substance (Fluorescein CI45350) is prepared and diluted with pure water. The fluorescent intensity must decrease with a diluted rate and it can be measured by a fluorescent photometer (ND3300, NanoDrop Inc). The dilution performance of each ladder is evaluated independently when the dilution rate is set to 10 times and the frequency of Braille pin at $6 \mathrm{~Hz}$. The reference liquid is diluted for 10 minutes for each dilution unit in order to get enough dilution time. Following to the each unit test, the 4 ladder dilution performance is evaluated continuously keeping each unit at 10 times dilution rate.

\section{Results \& Discussion}

4-1. Measurement of flow rate

Fig.8 shows the result of flow rate when the pumping frequency is kept between 3.13 to $6.0 \mathrm{~Hz}$ by the Braille device control program. From Fig.8 (A), it is known that the flow rate is proportioned to the pumping frequency and the maximum flow rate was $7.0 \mathrm{nl} / \mathrm{min}$ at $6 \mathrm{~Hz}$. As the flow rates measured either inside of micro-channel and outlet tends to same, only the inside of micro-channel flow rate is represented in the following experiments.Fig.8 (B) shows the effect of viscosity when changed between 1 to $7 \mathrm{mPa} \cdot \mathrm{s}$ that are almost similar to those of human saliva. As can be seen, the flow rate is inversely proportional to the viscosity. This result is quite theoretical because the higher viscosity results in a larger energy loss during flow as shown in the following equation (1).

$$
\mathrm{V}=\mathrm{UA}=\mathrm{Fh} / \mu
$$

Where $\mathrm{V}$ : flow rate, $\mathrm{U}$ : relative speed, $\mathrm{A}$ : cross section of channel, $\mathrm{F}$ : driving force, $\mathrm{h}$ : height of channel , $\mu$ : viscosity. As both of $\mathrm{F}$ and $\mathrm{h}$ constant, the flow rate $\mathrm{V}$ must be inverse proportional to the viscosity $\mu$.

(A)

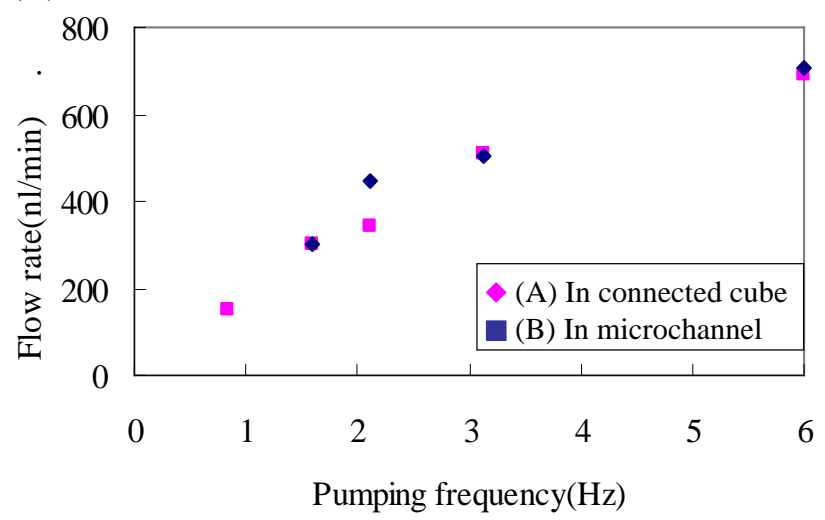

(B)

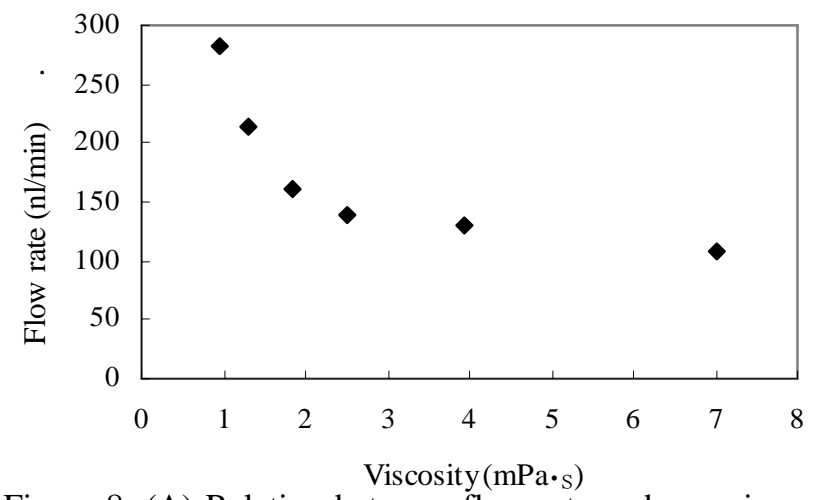

Figure 8: (A) Relation between flow rate and pumping frequency. (B) Relation between flow rate and viscosity. The pumping frequency was set to $3.13 \mathrm{~Hz}$.

\section{4-2. Evaluation of dilution performance}

Fig.9 shows the calibration curve when the reference liquid is diluted and the change of Relative Fluorescence Unit (RFU) is plotted, and it decreased logarithmically with the dilution rate. The dilution rate of diluted liquid through the 4 ladder dilution device is converted from this 
calibration curve.

Fig.10 (A) shows 10 times diluted results of each device, namely 4 steps in a ladder. The average remains in about 10 times as expected, however, the measured dilution rates scattered much lower than the target and the errors are about $\pm 20 \%$ of the targeted 10 times of dilution. Fig10. (B) shows the result of 4 ladder dilution systems. As easily forecasted, the error is accumurated with the dilution steps and the obtained dilution rates decreased with the steps against the target. Regrettably, the developed system is not enough for the practical application of saliva dilution systems for human stress diagnosis for the moment and the dilution accuracy must be improved.

The causes of dilution error are considered be caused by a pumping inaccuracy and evaporation of the tested liquid. The former can be improved by more accurate lithography of micro-channels fabrication and better control of Braille pin motion. The latter brought a new technological problem depending on material's characteristic of PDMS so as to absorb and evaporate water to some extent. The amount of evaporation was measured by the another test, where a chip of PDMS was put on an automatic balance under conditions of $24^{\circ} \mathrm{C}$ and $60 \%$ humidity and could verify that about $30 \%$ of water in micro-channel is evaporated within 10 minutes. PDMS is an excellent material to lithograph micro-channels easily at the laboratory, however, its inside surface must be coated by some suitable material so as to protect evaporation of water.

\section{Conclusion}

In the present paper, a new dilution system to dilute human's saliva step-likely at $10^{1}, 10^{2}, 10^{3}$ and $10^{4}$ times was proposed and its principle was verified by the test using the prototype of 4 ladder dilution system. The rate of dilution and flow were observed from the change of fluorescence intensity measured by the fluorescence photometer. Unfortunately, the diluted rates remained $\pm 20 \%$ of the target and difficult to apply it to the human stress diagnosis for the moment. The way how to improve the performance so as to be applicable in practice is also discussed.

\section{References}

[1] S. Wakida., Y. Tanaka., H. Nagai.,2004, Bunseki, pp309-316.

[2] H. Nakajima., H. Masuda., S. Ishino., T. akagama., T. Shimosaka., K.Arai., Y.Yoshimura., K.Uchiyama., 2005, BUNSEKI KAGAKU Vol.54, No.9, pp817-823.

[3] TAMA TLO Co, 2006, "The development of fast high throughput evaluation system for human fitness utilizing MEMS technology".

[4] Y.Tanaka., N. Naruishi., Y Nakayama., T. Higashi., S. WAkida., 2006, J. Chromatgr, A 1190, pp132-137.

[5] Y. Hioki., K. Morishima., K. Watanabe., Y. Furukawa., S. Takayama., 2006, ROBOMEC 2006 in WASEDA, Vol.2006 Page.1P1-C11.

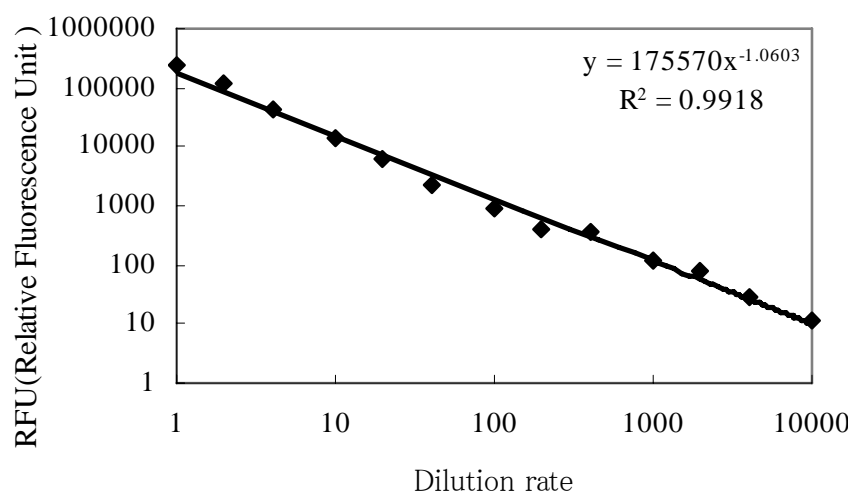

Figure 9: Calibration curve by fluorescent intensity. The dilution rate is calculated by this curve.

(A)

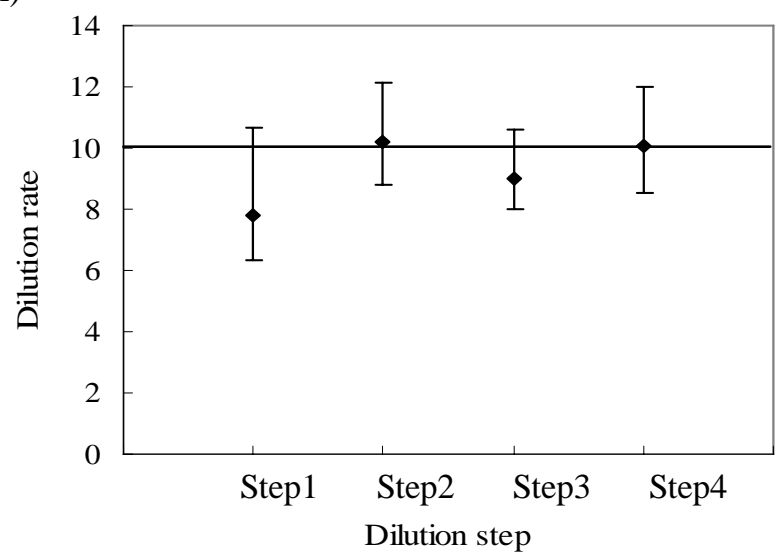

(B)

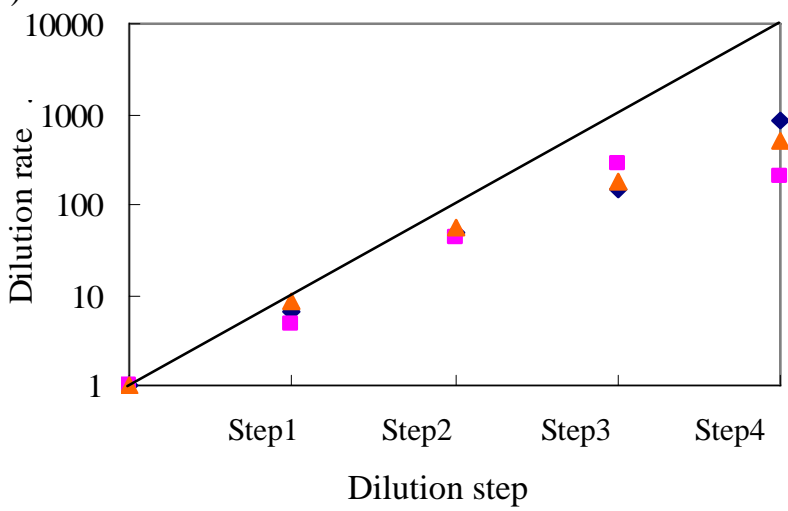

Figure 10: (A) Evaluation of the 10-fold dilution. The mean value and the error range are shown. (B) Evaluation of four ladder dilution. The dilution rate is set to $10^{4}$-fold totally, and the dilution rate of each stage was set to 10 -fold.

[6] T. Tomikawa., Y. Jou., K. Morishima., Y. Furukawa., K. Watanabe., S. Takayama., 2006, Proceeding of the 19th bio engineering conference, 2006 annual meeting of BED/JSME, pp466-467.

[7] W. Gu., X. Zhu., N. Futai., B. S. Cho., S. Takayama., 2004, PNAS, Vol.101, No. 45, 15861-15866.

[8] J. W. Song., W. Gu., N. Futai., K. A. Warner., J. E. Nor., Shuichi Takayama., 2005, Anal Chem Vol.77, No 13, 3993-3999. 